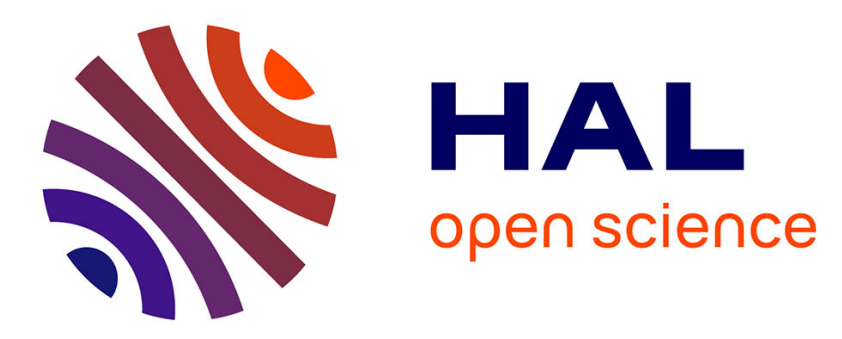

\title{
Adapting a memory fMRI research protocol in clinical routine: Feasibility and results
}

\author{
Ovidio Solano Cabrera, Stéphane Lehéricy, Véronique Masson, Séverine \\ Samson, Sophie Dupont
}

\section{- To cite this version:}

Ovidio Solano Cabrera, Stéphane Lehéricy, Véronique Masson, Séverine Samson, Sophie Dupont. Adapting a memory fMRI research protocol in clinical routine: Feasibility and results. Epilepsy \& Behavior, 2018, 81, pp.49-54. 10.1016/j.yebeh.2017.11.018. hal-01741854

\section{HAL Id: hal-01741854 https: / hal.sorbonne-universite.fr/hal-01741854}

Submitted on 23 Mar 2018

HAL is a multi-disciplinary open access archive for the deposit and dissemination of scientific research documents, whether they are published or not. The documents may come from teaching and research institutions in France or abroad, or from public or private research centers.
L'archive ouverte pluridisciplinaire HAL, est destinée au dépôt et à la diffusion de documents scientifiques de niveau recherche, publiés ou non, émanant des établissements d'enseignement et de recherche français ou étrangers, des laboratoires publics ou privés. 


\title{
Adapting a memory fMRI research protocol in clinical routine: Feasibility and results
}

\author{
Ovidio Solano Cabrera a,b ${ }^{\mathrm{a}}$, Stéphane Lehéricy ${ }^{\mathrm{c}, \mathrm{d}, \mathrm{e}}$, Véronique Masson ${ }^{\mathrm{a}}$, Séverine Samson ${ }^{\mathrm{f}}$, Sophie Dupont ${ }^{\mathrm{a}, \mathrm{d}, \mathrm{e}, \mathrm{g}, *}$ \\ ${ }^{a}$ Epilepsy unit, AP-HP Groupe hospitalier Pitié-Salpêtrière-Charles Foix, F-75013 Paris, France \\ b Epilepsy Clinic, Neurology department, Social Security Salvadoran Institute, San Salvador, El Salvador \\ c Neuroradiology unit, AP-HP Groupe hospitalier Pitié-Salpêtrière-Charles Foix, F-75013 Paris, France \\ d Inserm U 1127, CNRS UMR 7225, UMR S 1127, Institut du Cerveau et de la Moelle épinière - ICM, Centre de Neuroimagerie de Recherche - CENIR, F-75013 Paris, France \\ e Sorbonne University, UPMC Univ. Paris 06, F-75005 Paris, France \\ ${ }^{\mathrm{f}}$ Laboratoire PSITEC (EA 4072), Université de Lille, F-59000 Lille, France \\ ${ }^{g}$ Rehabilitation unit, AP-HP Groupe hospitalier Pitié-Salpêtrière-Charles Foix, F-75013 Paris, France
}

Keywords:

Medial temporal lobe epilepsy

fMRI

Episodic memory

Neuropsychological testing

Postoperative outcome

\begin{abstract}
A B S T R A C T
Objective: The objective of this study was to test the reliability of functional magnetic resonance imaging (fMRI) evaluation of memory function in clinical practice to predict postoperative memory decline in patients with refractory medial temporal lobe epilepsy (MTLE) candidate to surgery.

Methods: Twenty-six consecutive patients with MTLE who underwent a complete presurgical evaluation were included. All patients underwent fMRI memory study and complete neuropsychological assessment. Lesions consisted in hippocampal sclerosis in 18 patients (12 right and 6 left), dysembryoplastic neuroepithelial tumor (DNET) in 5 cases (4 right, 1 left), epidermoid cyst in one patient (right). Two patients had no lesion (2 left). Results: Nineteen patients (73\%) underwent surgery. The other seven patients (27\%) declined surgery, mainly because of the risk of memory deficit. The fMRI procedure correctly predicted both verbal and nonverbal memory postoperative outcome in 13 of the patients (72\%), failed to predict a postoperative memory worsening in only two patients (12\%), and predicted worsening in three patients (17\%) that remained stable (versus $44 \%, 39 \%$, and $17 \%$ with the sole neuropsychological testing). The reliability of the fMRI procedure was not influenced by the type of lesion, the side of the epileptic focus, or the type of preoperative memory profile (typical or atypical). Significance: Appearing as a valuable clinical tool to predict postoperative memory outcome, fMRI may add information over and above other available tests.
\end{abstract}

\section{Introduction}

Surgery is an effective therapy for refractory medial temporal lobe epilepsy (MTLE) [1]. Candidate patients with epilepsy to the surgery are usually concerned by the following two major points: i) seizure freedom after surgery and ii) after effects following surgery, especially the risk of memory decline. Indeed, a substantial number of patients with MTLE experience a worsening of episodic memory following surgery [2]. Several studies intended to identify clinical factors that are associated with a significant postoperative memory decline. These factors include surgery in the dominant hemisphere for language, low preoperative memory function, later age of seizure onset, low verbal IQ persistence of seizures after surgery, persistence of interictal epileptiform discharges on postoperative electroencephalography (EEG), postictal memory deficit, mood disturbance before surgery, and magnetic

* Corresponding author at: Epileptology Unit and Rehabilitation Unit, Hôpital de la Salpêtrière, 47, boulevard de l'Hôpital, 75651 Paris cedex 13, France.

E-mail address: sophie.dupont@aphp.fr (S. Dupont). resonance imaging (MRI) findings other than exclusively unilateral medial temporal sclerosis [2-13]. To date, the "gold standard" prediction of potential postoperative memory deficits is still based on neuropsychological measures and the Wada test. But recent studies have pointed out the interest of functional imaging studies to improve the prediction of postoperative memory outcome. Firstly, studies have demonstrated the ability of memory functional MRI (fMRI) protocols to replace the Wada test and accurately predict memory outcome [14-18]. Further studies have confirmed the potential of fMRI as a preoperative predictor of postsurgical memory decline. Most of these studies suggested that the functional capacity of the resected hippocampus, rather than the functional reserve of the contralateral hippocampus, was what determined the postoperative memory decline [19-25]. In other terms, patients with a consistent fMRI activation of the hippocampus to be resected were at high risk of postoperative memory decline. In 2010, our team has conducted a study to determine which of fMRI or Wada test were the best predictors of postoperative changes in memory and could help to provide to patients a precise counseling before surgery [26]. We found that the equation based on left fMRI 
medial temporal lobe (MTL) activation during delayed recognition, side of the epileptic focus and preoperative global verbal memory score correctly determined the worsening of verbal memory in $90 \%$ of the patients. Wada test data were not predictive of changes in either verbal or nonverbal memory. Based on these promising preliminary results, we decided to implant our research protocol in clinical routine in order to validate its feasibility and reliability. We wanted to test the reliability of our protocol research that had been validated in patients with MTLE with hippocampal sclerosis with atypical memory profiles in consecutive patients with MTLE with and without hippocampal sclerosis, with typical or atypical memory profiles. We also aimed to study the impact of the fMRI results on the surgical decision.

\section{Methods}

\subsection{Subjects}

The study population included 26 patients with consecutive MTLE who underwent a complete presurgical evaluation in our epilepsy center from September 2014 to September 2015.

This retrospective study was approved by the local ethics committee, in agreement with the Declaration of Helsinki. Informed written consent was obtained from all patients.

Inclusion criteria were the following:

1 Well explored unilateral MTLE with at least a prolonged surface video-EEG and a structural MRI;

2 Complete neuropsychological assessment including episodic verbal and visuospatial tests (Full Scale IQ (digit)-span, verbal memory testing (Jones-Gotman verbal learning (MJG) and/or Rey auditory verbal learning test) and nonverbal testing (Rey/Taylor complex figure, and/or Aggie's figures visual learning);

3 Decision of surgery for epileptic reasons subject to memory assessment.

The degree of deficit of learning and recognition in verbal and nonverbal memory for every patient was categorized as normal, slight, moderate, and severe.

\subsection{Protocol}

We used the fMRI memory protocol validated by our team [26]. Memory tasks included episodic memory encoding and recognition tasks. Patients are tested on two consecutive days to create two recognition conditions that differed in relation to the delay of recognition as follows: immediate recognition or 24-hour-delayed recognition. For each session, a sequential task-activation block paradigm was employed alternating an experimental condition and a control condition. Session 1 was performed outside the scanner.

In Session 1, the encoding task consisted of three blocks of 12 study stimuli repeated over the 3 blocks alternating with four blocks of 12 control stimuli. Stimuli consisted of color photographs of simple objects, such as fruit, flowers or animals that may be encoded by both verbal and nonverbal strategies. Each stimulus was presented during $3 \mathrm{~s}$. Each block lasted for $36 \mathrm{~s}$. Control stimuli were obtained by degrading study stimuli with a random-rectilinear algorithm from Adobe Photoshop 6.0. Patients were explicitly instructed to try to remember the studied stimuli for a later test.

After a delay of $60 \mathrm{~s}$, patients performed the recognition task. During this task, subjects were presented with 24 stimuli consisting in 12 novel stimuli and the 12 previously learnt stimuli, displayed in blocks of 8 stimuli ( 4 new/4 study) alternating with four blocks of 8 control stimuli. Each stimulus was presented during $3 \mathrm{~s}$. Each block lasted for $24 \mathrm{~s}$.

In Session 2, the following day, subjects began the fMRI session by a 24-hour-delayed recognition of stimuli encountered the previous day followed by new encoding and immediate recognition tasks.

\section{3. fMRI acquisition}

The MRI data were acquired from an MRI machine 3T GE Signa. The subjects were placed in supine position. Their heads were immobilized with cushions to reduce movement artifacts. The images were projected onto a mirror located at the end of the bore of the imager. For each subject, a classic structural T1-weighted three-dimensional (3D) Brain Volume imaging (BRAVO) was first collected to provide detailed anatomical information. Then functional imaging was performed using 39 continuous slices using single shot gradient echo planar imaging covering the entire brain in the coronal plane perpendicular to the long axis of the hippocampus (repetition time $=3 \mathrm{~s}$, echo time $=35 \mathrm{~ms}$, flip angle $=90^{\circ}$, matrix $=64 \times 64$, field of view $=20 \times 20 \mathrm{~cm}^{2}$, slice thickness $=3 \mathrm{~mm}$ ). The encoding task included 84 images (acquisition time $=4 \mathrm{~min}$ and $12 \mathrm{~s}$ ) and the delayed and immediate recognition tasks included 56 images (acquisition time $=2 \mathrm{~min}$ and $48 \mathrm{~s}$ ). Four dummy scans were added at the beginning of each fMRI acquisition. MRI acquisitions including both structural and functional images lasted $45 \mathrm{~min}$.

\subsection{Data analysis}

Data processing was performed using Brainwave software ${ }^{\circledR}$ based on an advantage workstation (General Electrics, Milwaukee). The fMRI scans were corrected for head movements using a sixparameter rigid body motion correction via registration to the first volume. The realigned functional images were realigned to the anatomical reference in the native space and smoothed with a $4-\mathrm{mm}$ Gaussian filter. General linear models were defined individually across runs, each with one regressor per condition. The task regressors were defined as a box-car convolved with the canonical hemodynamic response function. Voxels were considered to be significantly activated in comparison with the reference task at z-score $>3$, uncorrected for multiple comparisons. This individual analysis focused on MTL activations (hippocampus proper and parahippocampal cortices, Brodmann areas 27,28 , and 34-36). Only individual analysis was performed for each subject.

\subsection{1. fMRI lateralization}

To evaluate MTL lateralization, we considered the presence or absence of activation within the left and right hippocampal and parahippocampal regions. Images were visually inspected for activations in these areas as usually done during the regular neuroradiological reading in a clinical setting. Activation was considered lateralized in the right or left MTL if it was clearly predominant in one hemisphere and bilateral if activation was symmetrical or mildly asymmetric.

\subsection{Surgical decision}

After the neuropsychological and fMRI assessments, each patient was reviewed during the neurological staff to take the surgical decision according to both neuropsychological and fMRI activation data. The surgical indication was retained in case of the following:

1) perfect congruence of electroclinical data from video-EEG and MRI imaging

2) no risk of postoperative amnesia

When a memory decline was predictable, enlightened information was provided to the patient, so that he could make his decision.

\subsection{Postoperative evaluation}

One year after surgery, all patients were systematically hospitalized during 5 days to undergo a complete postoperative evaluation including clinical visit, MRI, EEG, and a neuropsychological assessment including 
episodic verbal and visuospatial tests (Full Scale IQ (digit)-span, verbal memory testing (Jones-Gotman verbal learning (MJG) and/or Rey auditory verbal learning test) and nonverbal testing (Rey/Taylor complex figure and/or Aggie's figures visual learning) that was identical to the assessment performed in the preoperative period.

\section{Results}

\subsection{Clinical data}

We included 26 patients ( 20 women and 6 men) with a mean age of $42 \pm 15$ years. Twenty-five patients were right-handed and one patient was left-handed; 17 patients had right MTLE and 9 left MTLE. Lesions consisted in hippocampal sclerosis in 18 patients (12 right and 6 left), dysembryoplastic neuroepithelial tumor (DNET) in 5 cases (4 right, 1 left), epidermoid cyst in one patient (right). Two patients had no lesion. (See Table 1.)

\subsection{Neuropsychological data}

The results of the neuropsychological tests showed that the patients had an average global intelligence quotient (GIQ) of $83 \pm 13$ (range: 61-115), a mean verbal intelligence quotient (VIQ) of $92 \pm 13$ (range: 65-112), and a mean performance intelligence quotient (PIQ) of $89 \pm 13$ (range: 64-116); for two patients IQ assessment was not possible because of the language barrier, those case were classified as "noncalculable" (Table 2).

Patients with right MTLE exhibited the following: i) nonverbal and verbal memory deficits in 14 patients (65\%), ii) exclusive verbal memory deficits in three patients (18\%), iii) exclusive nonverbal deficit in two patients (12\%), and iv) no deficit in one patient (6\%). Memory profile was typical (exclusive or predominant deficit of nonverbal memory) in only 5 patients (29\%) and atypical (no deficit, exclusive or

Table 1

Clinical data.

\begin{tabular}{|c|c|c|c|c|c|c|c|}
\hline $\mathrm{Pt}$ & Sex & $\begin{array}{l}\text { Hand } \\
\text { laterality }\end{array}$ & $\begin{array}{l}\text { Age } \\
\text { at } \\
\text { fMRI }\end{array}$ & Lesion & $\begin{array}{l}\text { Lesion } \\
\text { side }\end{array}$ & $\begin{array}{l}\text { Epileptic } \\
\text { focus } \\
\text { side }\end{array}$ & $\begin{array}{l}\text { Postoperative } \\
\text { sz outcome } \\
\text { (Engel's class) }\end{array}$ \\
\hline 1 & W & $\mathrm{R}$ & 26 & HS & $\mathrm{R}$ & $\mathrm{R}$ & II \\
\hline 2 & M & $\mathrm{R}$ & 25 & DNET & $\mathrm{R}$ & $\mathrm{R}$ & Ia \\
\hline 3 & $\mathrm{M}$ & $\mathrm{R}$ & 44 & HS & $\mathrm{L}$ & $\mathrm{L}$ & II \\
\hline 4 & W & $\mathrm{R}$ & 38 & No lesion & - & $\mathrm{L}$ & - \\
\hline 5 & M & $\mathrm{R}$ & 47 & HS & $\mathrm{L}$ & $\mathrm{L}$ & Ia \\
\hline 6 & W & $\mathrm{R}$ & 55 & $\begin{array}{l}\text { Epidermoid } \\
\text { cyst }\end{array}$ & $\mathrm{R}$ & $\mathrm{R}$ & - \\
\hline 7 & W & $\mathrm{R}$ & 25 & HS & $\mathrm{R}$ & $\mathrm{R}$ & Ia \\
\hline 8 & W & $\mathrm{R}$ & 30 & HS & $\mathrm{R}$ & $\mathrm{R}$ & Ia \\
\hline 9 & W & $\mathrm{R}$ & 42 & HS & $\mathrm{L}$ & $\mathrm{L}$ & ND \\
\hline 10 & W & $\mathrm{R}$ & 68 & HS & $\mathrm{L}$ & $\mathrm{L}$ & Ia \\
\hline 11 & $\mathrm{M}$ & $\mathrm{R}$ & 59 & HS & $\mathrm{R}$ & $\mathrm{R}$ & Ia \\
\hline 12 & $\mathrm{M}$ & $\mathrm{R}$ & 52 & HS & $\mathrm{R}$ & $\mathrm{R}$ & - \\
\hline 13 & W & $\mathrm{R}$ & 55 & HS & $\mathrm{R}$ & $\mathrm{R}$ & II \\
\hline 14 & W & $\mathrm{R}$ & 55 & HS & $\mathrm{R}$ & $\mathrm{R}$ & - \\
\hline 15 & W & $\mathrm{R}$ & 30 & HS & $\mathrm{R}$ & $\mathrm{R}$ & III \\
\hline 16 & W & $\mathrm{R}$ & 47 & HS & $\mathrm{R}$ & $\mathrm{R}$ & - \\
\hline 17 & W & $\mathrm{R}$ & 43 & DNET & $\mathrm{R}$ & $\mathrm{R}$ & - \\
\hline 18 & W & $\mathrm{R}$ & 57 & HS & $\mathrm{L}$ & $\mathrm{L}$ & - \\
\hline 19 & $\mathrm{M}$ & $\mathrm{R}$ & 32 & HS & $\mathrm{R}$ & $\mathrm{R}$ & Ia \\
\hline 20 & W & $\mathrm{R}$ & 46 & DNET & $\mathrm{R}$ & $\mathrm{R}$ & Ia \\
\hline 21 & W & $\mathrm{R}$ & 18 & DNET & $\mathrm{R}$ & $\mathrm{R}$ & Ia \\
\hline 22 & W & $\mathrm{R}$ & 25 & HS & $\mathrm{L}$ & $\mathrm{L}$ & Ia \\
\hline 23 & W & $\mathrm{R}$ & 49 & HS & $\mathrm{R}$ & $\mathrm{R}$ & II \\
\hline 24 & W & $\mathrm{R}$ & 45 & HS & $\mathrm{L}$ & $\mathrm{R}$ & Ia \\
\hline 25 & W & $\mathrm{R}$ & 31 & DNET & $\mathrm{L}$ & $\mathrm{L}$ & Ia \\
\hline 26 & $\mathrm{~W}$ & $\mathrm{~L}$ & 55 & No lesion & - & $\mathrm{L}$ & Ia \\
\hline
\end{tabular}

Pt: patient, M: man, W: woman, sz = seizure, HS: hippocampal sclerosis, DNET: dysembryoplastic neuroepithelial tumor, R: Right, L: Left.

Engel's class la = completely seizure-free since surgery, Engel's class II = rare disabling seizures ("almost seizure-free"), Engel's class III = worthwhile improvement. predominant deficit of verbal memory, equal deficit of verbal and nonverbal memory) in 12 patients (71\%).

Patients with left MTLE exhibited the following: i) verbal and nonverbal memory deficits in 9 patients (67\%) and ii) exclusive verbal memory deficits in 3 patients (33\%). Memory profiles were predominantly typical (exclusive or predominant deficit of verbal memory, $89 \%)$. Atypical memory profile was noticed in only one patient who exhibited a predominant deficit of nonverbal memory.

\section{3. fMRI data}

Patients with right MTLE exhibited the following: i) bilateral MTL activations in $82 \%$ with a right side predominance (ipsilateral) in $50 \%$, a left side predominance (contralateral) in $43 \%$, and no predominance in $7 \%$; ii) exclusive right MTL activation (ipsilateral) in 12\%; and iii) exclusive left MTL activation (contralateral) in 6\%.

Patients with left MTLE exhibited the following: i) bilateral MTL activations in $89 \%$ with a left (ipsilateral) side predominance in $37.5 \%$ and right (contralateral) side predominance in $62.5 \%$ and ii) exclusive left MTL activation in $11 \%$.

\subsection{Surgical decision}

The following are according to the neuropsychological preoperative results:

- Surgery was recommended without reservations for a possible postoperative worsening of episodic memory in fourteen patients (54\%) (all typical memory profiles).

- Surgery was recommended with a warning about a possible postoperative worsening of episodic memory in nine patients (35\%) (all atypical memory profiles).

- Surgery was discouraged due to a predictable major postoperative worsening of episodic memory in three patients (11\%) (all atypical memory profiles).

The following are according to the fMRI preoperative results:

- Surgery was recommended without reservations for a possible postoperative worsening of episodic memory in twelve patients (46\%) (typical in 5, atypical in 7).

- Surgery was recommended with a warning about a possible postoperative worsening of episodic memory in thirteen patients (50\%) (typical in 7, atypical in 6).

- Surgery was discouraged due to a predictable major postoperative worsening of episodic memory in one patient (4\%) (typical).

The neuropsychological and fMRI data were only congruent in eleven patients (42\%).

Finally, nineteen patients (73\%) have undergone surgery and seven patients (27\%) patients did not; one patient was rejected because of a major postoperative risk of memory decline according to fMRI; 4 patients declined surgery because of the memory risk according to fMRI, and two patients changed their mind and declined without specifying the reason. (See Table 3.)

\subsection{Postoperative outcome}

Concerning the postoperative seizure outcome, 13 patients (72\%) were completely seizure-free (Engel's class Ia); 4 patients had rare disabling seizures and were "almost seizure-free" (Engel's class II), and 1 patient had a worthwhile improvement (Engel's class III).

\subsection{Predictive value of neuropsychological and fMRI data}

Eighteen patients underwent a complete postoperative neuropsychological assessment one year after surgery, and one patient was 
Table 2

Preoperative neuropsychological test results.

\begin{tabular}{|c|c|c|c|c|c|c|c|c|c|c|}
\hline \multirow[t]{2}{*}{ Pt } & \multicolumn{3}{|l|}{ IQ } & \multirow[t]{2}{*}{ Focus side } & \multicolumn{2}{|c|}{ Verbal memory } & \multicolumn{2}{|c|}{ Nonverbal memory } & \multirow[t]{2}{*}{ Language } & \multirow[t]{2}{*}{ Executive functions } \\
\hline & GIQ & VIQ & PIQ & & Learning & Recognition & Learning & Recognition & & \\
\hline 1 & 85 & 85 & 84 & $\mathrm{R}$ & $\mathrm{N}$ & $\mathrm{N}$ & $\mathrm{N}$ & $\mathrm{N}$ & + & $\mathrm{N}$ \\
\hline 2 & 96 & 96 & 97 & $\mathrm{R}$ & $\mathrm{N}$ & $\mathrm{N}$ & $\mathrm{N}$ & ++ & $\mathrm{N}$ & $\mathrm{N}$ \\
\hline 3 & $\mathrm{NC}$ & NC & $\mathrm{NC}$ & $\mathrm{L}$ & +++ & +++ & ++ & ++ & ++ & + \\
\hline 4 & 92 & 92 & 94 & $\mathrm{~L}$ & $\mathrm{~N}$ & ++ & $\mathrm{N}$ & $\mathrm{N}$ & $\mathrm{N}$ & $\mathrm{N}$ \\
\hline 5 & 74 & 77 & 75 & $\mathrm{~L}$ & +++ & +++ & ++ & ++ & + & ++ \\
\hline 6 & 79 & 83 & 76 & $\mathrm{R}$ & +++ & + & + & ++ & + & ++ \\
\hline 7 & 88 & 103 & 71 & $\mathrm{R}$ & $\mathrm{N}$ & $\mathrm{N}$ & $\mathrm{N}$ & + & $\mathrm{N}$ & $\mathrm{N}$ \\
\hline 8 & 91 & 90 & 94 & $\mathrm{R}$ & ++ & ++ & ++ & ++ & $\mathrm{N}$ & + \\
\hline 9 & 61 & 65 & 64 & $\mathrm{~L}$ & +++ & +++ & ++ & ++ & ++ & ++ \\
\hline 10 & 101 & 104 & 97 & $\mathrm{~L}$ & +++ & ++ & + & + & + & ++ \\
\hline 11 & 115 & 111 & 116 & $\mathrm{R}$ & ++ & $\mathrm{N}$ & $\mathrm{N}$ & $\mathrm{N}$ & + & $\mathrm{N}$ \\
\hline 12 & 90 & 89 & 93 & $\mathrm{R}$ & +++ & ++ & ++ & ++ & ++ & ++ \\
\hline 13 & 92 & 102 & 80 & $\mathrm{R}$ & $\mathrm{N}$ & ++ & + & ++ & $\mathrm{N}$ & $\mathrm{N}$ \\
\hline 14 & 70 & 73 & 75 & $\mathrm{R}$ & +++ & ++ & +++ & ++ & +++ & ++ \\
\hline 15 & 103 & 100 & 107 & $\mathrm{R}$ & ++ & + & + & $\mathrm{N}$ & + & $\mathrm{N}$ \\
\hline 16 & 83 & 83 & 84 & $\mathrm{R}$ & +++ & +++ & $\mathrm{N}$ & $\mathrm{N}$ & + & ++ \\
\hline 17 & 98 & 100 & 98 & $\mathrm{R}$ & + & ++ & $\mathrm{N}$ & $\mathrm{N}$ & + & + \\
\hline 18 & 83 & 84 & 83 & $\mathrm{~L}$ & + & ++ & + & + & + & ++ \\
\hline 19 & $\mathrm{NC}$ & NC & 88 & $\mathrm{R}$ & $\mathrm{N}$ & ++ & $\mathrm{N}$ & + & + & + \\
\hline 20 & 108 & 112 & 98 & $\mathrm{R}$ & $\mathrm{N}$ & + & + & ++ & $\mathrm{N}$ & + \\
\hline 21 & 92 & 87 & 100 & $\mathrm{R}$ & + & ++ & $\mathrm{N}$ & + & + & $\mathrm{N}$ \\
\hline 22 & 86 & 96 & 72 & $\mathrm{~L}$ & + & $\mathrm{N}$ & $\mathrm{N}$ & $\mathrm{N}$ & + & ++ \\
\hline 23 & 108 & 107 & 104 & $\mathrm{R}$ & + & $\mathrm{N}$ & $\mathrm{N}$ & ++ & $\mathrm{N}$ & $\mathrm{N}$ \\
\hline 24 & 81 & 77 & 91 & $\mathrm{R}$ & ++ & ++ & + & + & + & $\mathrm{NC}$ \\
\hline 25 & 108 & 108 & 106 & $\mathrm{~L}$ & $\mathrm{~N}$ & + & $\mathrm{N}$ & $\mathrm{N}$ & $\mathrm{N}$ & $\mathrm{N}$ \\
\hline 26 & 88 & 86 & 93 & $\mathrm{~L}$ & + & + & ++ & ++ & + & ++ \\
\hline
\end{tabular}

Pt: patient, N: normal, + : slight deficit, ++ : moderate deficit, +++ : severe deficit, NC: noncalculable.

lost on follow-up. Eleven patients out of the 18 patients had a typical memory profile.

Ten patients exhibited an overall stabilization or improvement of their postoperative memory functioning. Postoperative memory worsening in the verbal memory domain was observed in one patient, the nonverbal memory in 4 patients, and both the verbal and nonverbal memory in 3 patients. Among the patients who exhibited a postoperative memory worsening, there was a majority of patients with right TLE ( 5 out of 8 patients), but we cannot conclude on the effect of the side of surgery since a higher proportion of patients with right

Table 3

Surgical decision.

\begin{tabular}{|c|c|c|c|c|c|c|c|}
\hline Patient & Lesion & Focus side & $\begin{array}{l}\text { Neuropsychological } \\
\text { memory deficit }\end{array}$ & $\begin{array}{l}\text { Memory fMRI } \\
\text { activation }\end{array}$ & $\begin{array}{l}\text { Decision for surgery according } \\
\text { to neuropsychological tests }\end{array}$ & $\begin{array}{l}\text { Decision for surgery } \\
\text { according to fMRI }\end{array}$ & $\begin{array}{l}\text { Patient's decision } \\
\text { for surgery }\end{array}$ \\
\hline 1 & HS & $\mathrm{R}$ & No & left $>$ right & No & Yes & Yes \\
\hline 2 & DNET & $\mathrm{R}$ & Nonverbal & right $>$ left & Yes & Caution & Yes \\
\hline 3 & HS & $\mathrm{L}$ & Verbal > nonverbal & right $>$ left & Yes & Yes & Yes \\
\hline 4 & No lesion & $\mathrm{L}$ & Verbal & left $>$ right & Yes & Caution & $\mathrm{No}^{\mathrm{a}}$ \\
\hline 5 & HS & $\mathrm{L}$ & Verbal > nonverbal & right $>$ left & Yes & Yes & Yes \\
\hline 6 & Epidermoid cyst & $\mathrm{R}$ & Verbal > nonverbal & bilateral & Caution & Caution & $\mathrm{No}^{\mathrm{b}}$ \\
\hline 7 & HS & $\mathrm{R}$ & Nonverbal & right $>$ left & Yes & Caution & Yes \\
\hline 8 & HS & $\mathrm{R}$ & Verbal > nonverbal & left $>$ right & Caution & Yes & Yes \\
\hline 9 & HS & $\mathrm{L}$ & Verbal > nonverbal & right $>$ left & Yes & Yes & Yes \\
\hline 10 & HS & $\mathrm{L}$ & Verbal > nonverbal & left $>$ right & Yes & Caution & Yes \\
\hline 11 & HS & $\mathrm{R}$ & Verbal & right $>$ left & Caution & Caution & Yes \\
\hline 12 & HS & $\mathrm{R}$ & Verbal > nonverbal & right $>$ left & Caution & Caution & $\mathrm{No}^{\mathrm{a}}$ \\
\hline 13 & HS & $\mathrm{R}$ & Nonverbal > verbal & left & Yes & Yes & Yes \\
\hline 14 & HS & $\mathrm{R}$ & Verbal $=$ nonverbal & right $>$ left & Caution & Caution & $\mathrm{No}^{\mathrm{a}}$ \\
\hline 15 & HS & $\mathrm{R}$ & Verbal > nonverbal & left $>$ right & Caution & Yes & Yes \\
\hline 16 & HS & $\mathrm{R}$ & Verbal & right & No & Caution & $\mathrm{No}^{\mathrm{a}}$ \\
\hline 17 & DNET & $\mathrm{R}$ & Verbal & left $>$ right & No & Yes & $\mathrm{No}^{\mathrm{b}}$ \\
\hline 18 & HS & $\mathrm{L}$ & Verbal > nonverbal & left & Yes & No & $\mathrm{No}^{\mathrm{C}}$ \\
\hline 19 & HS & $\mathrm{R}$ & Verbal > nonverbal & right $>$ left & Caution & Caution & Yes \\
\hline 20 & DNET & $\mathrm{R}$ & Nonverbal > verbal & right $>$ left & Yes & Caution & Yes \\
\hline 21 & DNET & $\mathrm{R}$ & Verbal > nonverbal & left $>$ right & Caution & Yes & Yes \\
\hline 22 & HS & $\mathrm{L}$ & Verbal & left $>$ right & Yes & Caution & Yes \\
\hline 23 & HS & $\mathrm{R}$ & Nonverbal > verbal & right & Yes & Caution & Yes \\
\hline 24 & HS & $\mathrm{R}$ & Verbal > nonverbal & left $>$ right & Caution & Yes & Yes \\
\hline 25 & DNET & $\mathrm{L}$ & Verbal & right $>$ left & Yes & Yes & Yes \\
\hline 26 & No lesion & $\mathrm{L}$ & Nonverbal > verbal & right $>$ left & Yes & Yes & Yes \\
\hline
\end{tabular}

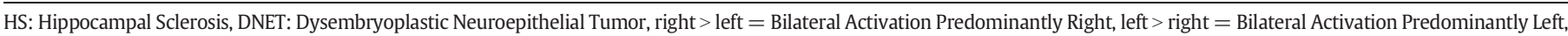
bilateral = Bilateral Activation without Predominance, $\mathrm{sz}=$ seizure, ND: not done (lost of view).

a Declined because of memory risk.

b Changed her mind.

c Rejected by the medical staff. 
Table 4

Postoperative neuropsychological results and predictive value of preoperative neuropsychological and fMRI data.

\begin{tabular}{|c|c|c|c|c|c|c|c|c|c|c|}
\hline \multirow[t]{2}{*}{ Pt } & \multirow{2}{*}{$\begin{array}{l}\text { Focus } \\
\text { side-lesion }\end{array}$} & \multicolumn{2}{|c|}{ Verbal memory } & \multicolumn{2}{|c|}{ Nonverbal memory } & \multirow[t]{2}{*}{ Language } & \multirow{2}{*}{$\begin{array}{l}\text { Executive } \\
\text { functions }\end{array}$} & \multirow{2}{*}{$\begin{array}{l}\text { Memory } \\
\text { profile }\end{array}$} & \multirow{2}{*}{$\begin{array}{l}\text { Prediction according } \\
\text { to neuropsychological } \\
\text { tests }\end{array}$} & \multirow{2}{*}{$\begin{array}{l}\text { Prediction } \\
\text { according } \\
\text { to fMRI }\end{array}$} \\
\hline & & Learning & Recognition & Learning & Recognition & & & & & \\
\hline 1 & R-HS & $S$ & $S$ & $\mathrm{~S}$ & $\mathrm{~S}$ & I & $\mathrm{S}$ & atypical & WWP & correct \\
\hline 2 & R-DNET & $\mathrm{S}$ & $\mathrm{S}$ & $\mathrm{S}$ & I & $\mathrm{S}$ & $\mathrm{S}$ & typical & correct & WWP \\
\hline 3 & L-HS & $\mathrm{S}$ & $\mathrm{S}$ & $\mathrm{S}$ & $\mathrm{S}$ & W & $\mathrm{S}$ & typical & correct & correct \\
\hline 5 & L-HS & $S$ & W & I & $\mathrm{S}$ & $S$ & I & typical & WSP & WSP \\
\hline 7 & R-HS & $\mathrm{S}$ & $\mathrm{S}$ & $\mathrm{S}$ & I & $\mathrm{S}$ & $\mathrm{S}$ & typical & correct & WWP \\
\hline 8 & R-HS & $S$ & $\mathrm{~S}$ & W & W & $\mathrm{N}$ & I & atypical & correct & WSP \\
\hline 10 & L-HS & W & W & W & W & W & W & typical & WSP & correct \\
\hline 11 & R-HS & W & W & W & W & $\mathrm{S}$ & I & atypical & WSP & correct \\
\hline 13 & R-HS & I & I & I & $S$ & $S$ & $S$ & typical & correct & correct \\
\hline 15 & R-HS & $\mathrm{S}$ & $\mathrm{S}$ & $\mathrm{S}$ & $\mathrm{S}$ & $\mathrm{S}$ & $\mathrm{S}$ & atypical & WSP & WWP \\
\hline 19 & R-HS & $S$ & I & $\mathrm{S}$ & W & $\mathrm{S}$ & I & atypical & correct & correct \\
\hline 20 & R-DNET & $S$ & $S$ & W & W & $S$ & $S$ & typical & WSP & correct \\
\hline 21 & R-DNET & $\mathrm{S}$ & I & $\mathrm{S}$ & $\mathrm{S}$ & I & $\mathrm{S}$ & atypical & WWP & correct \\
\hline 22 & L-HS & I & $S$ & W & W & W & W & typical & WSP & correct \\
\hline 23 & R-HS & W & W & W & W & $S$ & $S$ & typical & WSP & correct \\
\hline 24 & R-HS & $S$ & $S$ & $S$ & $S$ & $S$ & $S$ & atypical & WWP & correct \\
\hline 25 & L-DNET & $\mathrm{S}$ & $S$ & $S$ & $S$ & $\mathrm{~S}$ & $S$ & typical & correct & correct \\
\hline 26 & L-no & I & I & I & I & W & W & typical & correct & correct \\
\hline
\end{tabular}

$\mathrm{S}=$ stable, $\mathrm{I}=$ improvement, $\mathrm{W}=$ worsening, $\mathrm{ND}=$ not determined, WSP = wrong stabilization prediction, WWP = wrong worsening prediction.

TLE underwent surgery (67\%). The type of lesion or the side of the epileptic focus did not influence the postoperative memory functioning. (See Table 4.)

Of the 18 patients who underwent a complete neuropsychological assessment:

- Correct postoperative memory outcome prediction was depicted in 8 patients (44\%) with the preoperative neuropsychological assessment (typical memory profile in $75 \%$ ) and in 13 patients (72\%) with the fMRI procedure (typical memory profile in 62\%).

- Wrong postoperative memory stabilization prediction was depicted in 7 patients (39\%) with the preoperative neuropsychological assessment (typical memory profile in 66\%) and in 2 patients (12\%) with the fMRI procedure (typical memory profile in $50 \%$ ).

- Wrong postoperative memory worsening prediction was depicted in 3 patients (17\%) with the preoperative neuropsychological assessment (all atypical memory profiles) and in 3 patients (17\%) with the fMRI procedure (typical memory profile in 66\%).

\section{Discussion}

The following three major results emerged from this real life study: i) an fMRI procedure may reliably predict postoperative memory outcome in everyday practice; ii) the fMRI procedure was reliable whatever the type of lesion, the side of the focus or the type of memory profile (typical or atypical), and iii) the fMRI procedure influenced the surgery decision since a significant proportion of patients declined surgery when the results of the examinations were communicated to them.

\subsection{Accurate prediction of postoperative memory outcome}

\subsection{1. fMRI}

Six studies have compared fMRI and Wada test with variable results. Two studies found a good agreement between the two techniques, but these studies were performed on small groups of patients $[14,15]$. The four other studies did not find a very good agreement between the two modalities [16-18,26]. Several other functional neuroimaging studies have assessed the postoperative memory prediction, independently of the Wada test procedure, with a good correspondence between functional imaging predictions and postoperative real memory outcome. Richardson et al. have demonstrated in patients with LTLE that a relatively greater verbal memory encoding activity in the left hippocampus was the best predictor of the verbal memory decline after surgery $[19,20]$. Similarly, this time in patients with RTLE, Jansky et al. have shown that reduced activation of the medial temporal lobe region ipsilateral to the epileptogenic region correlated with a favorable memory outcome [21]. Powell et al. studied 15 patients with MTLE (8 left/7 right) and demonstrated that patients with relatively greater ipsilateral compared to contralateral medial temporal lobe activation had greater memory decline following surgery, both for verbal and nonverbal memory [22]. Frings et al. have shown that lateralization of hippocampal activation was significantly correlated with decline in verbal learning after surgery in a group of 22 patients with epilepsy [23]. Similarly, Bonelli et al. demonstrated that in left temporal lobe epilepsy, greater left than right anterior hippocampal activation on word encoding correlated with greater verbal memory decline after left anterior temporal lobe resection, while greater left than right posterior hippocampal activation correlated with better postoperative verbal memory outcome $[24,25]$.

Most of these fMRI studies that examined the postoperative memory outcome concerned patients with MTLE with hippocampal sclerosis.

\subsubsection{Type of lesion}

In our study, patients with MTLE without lesion or with lesions other than hippocampal sclerosis, i.e., DNET, had a reliable fMRI prediction of postoperative memory outcome in $80 \%$ of cases, whereas patients with MTLE with hippocampal sclerosis had a reliable fMRI prediction of postoperative memory outcome in $69 \%$ of cases.

\subsubsection{Side of focus and memory profiles}

Traditionally, left hippocampal impairment is associated with a deficit of verbal episodic memory, whereas right hippocampal impairment is associated with nonverbal episodic memory deficits [2]. Based on this model, preoperative memory ability is considered as the best predictor of postoperative memory decline in patients with MTLE candidates to surgery [8]. Our data, as other recent data, question the validity of this classical approach based on a material-specific lateralization of memory function. A recent meta-analysis of memory deficits after resection of left or right anterior temporal lobe has shown that impairment of verbal memory was consistently associated with the resection of the left dominant temporal lobe, whereas nonverbal deficits were less reliably observed after resection of the right temporal lobe and were frequently reported after either the right or the left temporal lobe resection [3]. Interestingly, in our study, the vast majority of our patients exhibited preoperatively both verbal and nonverbal episodic memory whatever the side of the focus and the type of lesion. Patients with right MTLE were more prone to have atypical memory profiles 
(no deficit, exclusive or predominant deficit of verbal memory, equally deficit of verbal and nonverbal memory depicted in $71 \%$ of patients with right MTLE), whereas all but one patient with left MTLE exhibited a deficit of verbal memory (predominantly or exclusively). Moreover, the prediction of postoperative memory outcome based on the sole neuropsychological preoperative neuropsychological deficits was rather low: $44 \%$ of correct predictions as compared to the prediction of fMRI (72\%).

\subsection{Which indications for the fMRI procedure?}

The fMRI procedure correctly predicted both verbal and nonverbal memory postoperative outcome in $72 \%$ of patients and failed to predict a postoperative memory worsening in only $12 \%$ of patients (worsening of verbal memory in one patient and of nonverbal memory in another patient). The question is now to determine if the fMRI procedure should be limited to patients with atypical memory profiles or systematically performed in addition to preoperative neuropsychological testing to improve the accuracy of preoperative information. Our data suggest that a preoperative atypical memory profile is not predictive of a worse memory outcome. Furthermore the fMRI procedure had the same reliability to predict the memory outcome in those patients with a typical memory profile (73\%) or atypical memory profile (71\%). In contrast, a typical memory profile was not predictive of a good memory outcome: $45 \%$ of patients with a typical preoperative memory profile exhibited a postoperative memory worsening, whereas $43 \%$ of patients with an atypical preoperative memory profile exhibited a postoperative memory worsening. These results suggest that the systematic performance of a preoperative memory fMRI besides the neuropsychological tests would allow improving the prediction of the postoperative memory deficit.

\subsection{Limitations of the study}

Results of the preoperative fMRI led to contraindication of the intervention in one patient, and communication of the fMRI results to the patients led six of them to refuse the intervention, mainly for fear of a postoperative worsening of the memory. It is therefore impossible to verify in these patients whether the prediction of worsening was correct.

Data analysis was performed by visual reading in a neuroradiological setting using clinical software provided by the vendor and nonstandardized statistical threshold for activation detection. It is possible that the use of a more standardized software such as SPM for data analysis combined with quantification of an asymmetry index as previously done may have improved the diagnostic performance of the memory fMRI procedure [26]. However, such software is not used in clinical practice.

\section{Conclusion}

It is now assumed that the advent of noninvasive functional neuroimaging has raised the possibility of replacing the old gold standards as the Wada test in the prediction of postoperative memory outcome. The efficacy of the Wada procedure in predicting memory outcome appeared limited, and there was no justification any more in considering them trustworthier than the data provided by neuroimaging [27]. Our study confirms that the fMRI procedure is reliable whatever the type of the lesion or the side of the epileptic focus. Appearing as a valuable clinical tool, fMRI may add information over and above other available tests and could be one of the tool to try to predict neuropsychological outcome.

\section{Conflict of interest statement}

The authors have no conflict of interest to disclose.

This research did not receive any specific grant from funding agencies in the public, commercial, or not-for-profit sectors.

\section{References}

[1] Dupont S, Tanguy M, Clemenceau S, Adam C, Hazemann P, Baulac M. Long-term prognosis and psychosocial outcomes after surgery for MTLE. Epilepsia 2006;47: 2115-24.

[2] Dupont S. Imaging memory and predicting postoperative memory decline in temporal lobe epilepsy: insights from functional imaging. Rev Neurol (Paris) 2015;171(3): 307-14.

[3] Lee TMC, Yip JTH, Jones-Gotman M. Memory deficits after resection from left or right anterior temporal lobe in humans: a meta-analytic review. Epilepsia 2002;43(3): 283-91.

[4] Chelune C. Hippocampal adequacy versus functional reserve: predicting memory functions following temporal lobectomy. Arch Clin Neuropsychol 1995;10(5): 413-32.

[5] Hemstaedter C. In: Sutula T, Pitkanen A, editors. Effects of chronic epilepsy on declarative memory systems. Elsevier; 2002. p. 439-53.

[6] Hermann BP, Wyler AR, Somes G, Dohan Jr FC, Berry III ADB, Clement L. Declarative memory following anterior temporal lobectomy in humans. Behav Neurosci 1994; 108:3-10.

[7] LoGalbo A, Sawrie S, Roth DL, Kuzniecky R, Knowlton R, Faught E, et al. Verbal memory outcome in patients with normal preoperative verbal memory and left medial temporal sclerosis. Epilepsy Behav 2005;6:337-41.

[8] Baxendale S, Thompson PJ, Sander JW. Neuropsychological outcomes in epilepsy surgery patients with unilateral hippocampal sclerosis and good preoperative memory function. Epilepsia 2013;54(9):131-4.

[9] Baxendale S, Thompson P, Harkness W, Duncan J. Predicting memory decline following epilepsy surgery: a multivariate approach. Epilepsia 2006;47:1887-94.

[10] Wisniewski I, Wendling AS, Steinhoff BJ. Impact of side of lesion, seizure outcome and interictal epileptiform discharges on attention and memory after surgery in TLE. Epileptic Disord 2011;13(1):27-35.

[11] Vulliemoz S, Prilipko O, Herrmann FR, Pollo C, Landis TPegna AJ, Seeck M. Can postictal memory predict postoperative memory in patients with TLE? Epilepsia 2012;53(10):170-e173.

[12] Stroup E, Langfitt J, Berg M, McDermott M, Pilcher, Como P. Predicting verbal memory decline following anterior temporal lobectomy (ATL). Neurology 2003; 60:1266-73

[13] Busch RM, Dulay MF, Kim KH, Chapin JS, Jehi L, Kalman CC, et al. Pre-surgical mood predicts memory decline after anterior temporal lobe resection for epilepsy. Arch Clin Neuropsychol 2001;26:739-45.

[14] Detre JA, Maccotta L, King D, Alsop DC, Glosser G, D'Esposito M, et al. Functional MRI lateralization of memory in temporal lobe epilepsy. Neurology 1998;50: 926-32.

[15] Golby A, Poldrack R, Illes J, Chen D, Desmond J, Gabrieli J. Memory lateralization in medial temporal lobe epilepsy assessed by functional MRI. Epilepsia 2002;43: 855-63.

[16] Killgore W, Glosser G, Casasanto D, French J, Alsop D, Detre J. Functional MRI and the Wada test provide complementary information for predicting post-operative seizure control. Seizure 1999;8:450-5

[17] Deblaere K, Backes W, Tieleman A, Vandemaele P, Defreyne L, Vonck K, et al Lateralized anterior mesiotemporal lobe activation: semirandom functional MR imaging encoding paradigm in patients with temporal lobe epilepsy - initial experience. Radiology 2005;236:996-1003.

[18] Rabin M, Narayan V, Kimberg D, Casasanto D, Glosser G, Tracy J, et al. Functional MR predicts post-surgical memory following temporal lobectomy. Brain 2004;127: 2286-98.

[19] Richardson M, Strange B, Duncan J, Dolan R. Memory fMRI in left hippocampal sclerosis optimizing the approach to predicting postsurgical memory. Neurology 2006;66:699-705.

[20] Richardson M, Strange B, Thompson P, Baxendale S, Duncan J, Dolan R. Pre-operative verbal memory fMRI predicts post-operative memory decline after left temporal lobe resection. Brain 2004;127:2419-26.

[21] Janszky J, Jokeit H, Kontopoulou K, Mertens M, Ebner A, Pohlmann-Eden B, et al. Functional MRI predicts memory performance after right mesiotemporal epilepsy surgery. Epilepsia 2005;46:244-50.

[22] Powell H, Richardson M, Symms M, Boulby P, Thompson P, Duncan J, et al. Preoperative fMRI predicts memory decline following anterior temporal lobe resection. J Neurol Neurosurg Psychiatry 2008;79:686-93.

[23] Frings L, Wagner K, Halsband U, Schwarzwald R, Zentner J, Schulze-Bonhage A Lateralization of hippocampal activation differs between left and right tempora lobe epilepsy patients and correlates with postsurgical verbal learning decrement. Epilepsy Res 2008;78:161-70.

[24] Bonelli SB, RHW Powell, Yogarajah M, Samson RS, Symms MR, Thompson PJ, et al. Imaging memory in TLE: predicting the effects of temporal lobe resection. Brain 2010:1-14.

[25] Bonelli SB, Thompson PJ, Yogarajah M, Powell RHW, Samson RS, McEvoy AW, et al Memory reorganization following anterior temporal lobe resection: a longitudinal functional MRI study. Brain 2013;136:1889-900.

[26] Dupont S, Duron E, Samson S, Denos M, Volle E, Delmaire C, et al. Functional MR imaging or Wada test: which is the better predictor of individual postoperative memory outcome? Radiology 2010;255:128-34.

[27] Papanicolaou AC, Rezaie R, Narayana S, Choudhri AF, Wheless JW, Castillo EM, et al. Is it time to replace the Wada test and put awake craniotomy to sleep? Epilepsia 2014;55:629-32. 